\title{
Isótopos Estables en Camélidos del Período Tardío del Noroeste de Argentina (NOA): Estrategias de Uso y Manejo de Rebaños
}

\author{
Stable Isotopes in Camelids from the Late Period of Northwest Argentina (NOA): Use \\ Strategies and Herd Management.
}

Guillermo Luis Mengoni Goñalons ${ }^{i}$

\section{RESUMEN}

Se presentan los resultados de isótopos estables $\left(\delta^{13} \mathrm{C}\right.$ y $\left.\delta^{15} \mathrm{~N}\right)$ realizados sobre diferentes huesos apendiculares $(n=28)$ de camélidos de tres sitios del Noroeste de Argentina (NOA) que fueron ocupados en tiempos tardíos-incaicos: Esquina de Huajra y Pucará de Volcán (Quebrada de Humahuaca, Jujuy) y Tolombón (Valles Calchaquíes, Salta). A partir de esta información se discuten los modos de acceso y redistribución de estos ungulados, tanto silvestres como domesticados. Los resultados alcanzados posibilitan ampliar el conocimiento que actualmente tenemos acerca de la complejidad de los sistemas de uso y manejo de estos animales que fueron fundamentales para las sociedades andinas antes de la etapa colonial.

Palabras Claves: Camélidos, Osteometría, Isótopos, Manejo

\section{ABSTRACT}

The stable isotope $\left(\delta^{13} \mathrm{C}\right.$ y $\left.\delta^{15} \mathrm{~N}\right)$ results on appendicular camelid bones $(n=28)$ from three sites in Northwestern Argentina are presented: Esquina de Huajra and Pucará de Volcán in the Quebrada de Humahuaca (Jujuy province) together with Tolombón in the Calchaquí Valley (Salta province) were occupied during both the Late and Incaic period. We discuss procurement and redistribution of both wild and domestic ungulates. The isotopic results allow us to broaden our present understanding of the complex camelid management system implemented by Andean societies in pre-Colonial times.

Key Words: Camelids, Osteometry, Isotopes, Management

i Instituto de Arqueología, Facultad de Filosofía y Letras, Universidad de Buenos Aires, 25 de Mayo 217 piso 3, 1002 Ciudad Autónoma de Buenos Aires, Argentina. Correo-e: wmengoni@yahoo.com.ar.

Recibido: 05-12-2012 Revisado: 05-05-2013 Aceptado: 20-12-2013 


\section{INTRODUCCIÓN}

En los Andes Centro-Sur se ha avanzado en los estudios de isótopos estables, especialmente en relación con la dieta humana. Más recientemente, se ha comenzado a trabajar con la dieta de aquellos recursos que han integrado la alimentación humana en esa región, tal es el caso de los camélidos (e.g., Dantas 2012, lzeta et al. 2009, López et al. 2012, Mengoni Goñalons 2007, Thornton et al. 20 I0).

Pese a ello aún no se ha explotado todo el potencial que tienen estos indicadores en los camélidos. Conocer la variabilidad de su dieta permite no sólo entender la dieta humana, sino discutir las prácticas de uso y manejo de estos animales, sean silvestres o domesticados. Presentamos los resultados obtenidos en el análisis osteométrico y de isótopos realizados sobre huesos de camélidos de tres sitios del periodo Tardío-Incaico del Noroeste de la Argentina (NOA): Esquina de Huajra a $2.000 \mathrm{~m}$ de altitud y Pucará de Volcán a $2.100 \mathrm{~m}$ (Quebrada de Humahuaca, Jujuy) y Tolombón a 1.700 m (Valles Calchaquíes, Salta).

\section{VARIABILIDAD ISOTÓPICA EN LOS CAMÉLIDOS ACTUALES DEL NOA}

La información isotópica actualmente disponible para los camélidos actuales del NOA posibilita plantear varias cuestiones de interés general. A los primeros trabajos (e.g, Fernández y Panarello 1999-200I) se han sumado nuevos aportes que amplían el conocimiento disponible (e.g., Yacobaccio et al. 20l0).

Los estudios isotópicos realizados con muestras de camélidos modernos mostraron inicialmente diferencias significativas entre los valores del $\delta^{13} \mathrm{C}$ para los animales que viven a más de $4.000 \mathrm{~m}$, con respecto de aquellos que se alimentan a menor altitud (e.g., Panarello y Fernández 2002). Esto se debería a que las pasturas (hierbas y gramíneas) que estos herbívoros usan en cada zona altitudinal están compuestas por porcentajes diferentes de plantas con distinto camino fotosintético: $\mathrm{C}_{3}(-29.1 \%$ 。 a $-24 \%$ ), $C_{4}(-13.8 \%$ a $-10.6 \%$ o o su combinación (Fernández y Panarello 1999-200I).

Los camélidos que viven a más de $4.000 \mathrm{~m}$ muestran una dieta dominada por plantas $\mathrm{C}_{3}$ y los que viven a menos de $4.000 \mathrm{~m}$ poseen una dieta mixta $\mathrm{C}_{3}$ y $\mathrm{C}_{4}$, según Fernández y Panarello (I999-200I). Los trabajos más recientes realizados en llamas y vicuñas que viven en la puna argentina entre 3600 y 4700 m fortalecen esa tendencia que muestra que tanto los camélidos domesticados como los silvestres criados en el pajonal se alimentan de $\mathrm{C}_{3}$ (Poa sp y Festuca $\mathrm{sp})$, y que los que frecuentan el tolar incorporan a su dieta plantas $\mathrm{C}_{4}$, al igual que en las vegas (Yacobaccio et al. 20l0).A su vez, los herbívoros que se alimentan de estas plantas enriquecen sus tejidos en un $5 \%$, haciéndose sus lecturas más positivas (Fernández y Panarello 1999-200I).

Los datos recopilados por estos estudios muestran que los camélidos actuales que viven a más altura $(>4.000 \mathrm{~m})$ exhiben valores medios más bajos (guanaco: -19,4\%o; vicuña: -18,3\%; llama: -19,3\%o) que aquellos que se alimentan a menor altura (vicuña: -15,3\%o; llama: - I7,0\%). Cabe señalar que para su comparación con las muestras arqueológicas los valores actuales recién mencionados fueron corregidos (e.g., Yacobaccio et al. 2010) para compensar el "efecto Suess", sumándose un I,5\%。 (Pate 1994). Estos valores serán tomados de referencia cuando discutamos los resultados de los isótopos en muestras arqueológicas.

\section{SELECCIÓN DE LA MUESTRAY SU VARIABILIDAD OSTEOMÉTRICA}

Los materiales óseos fueron identificados anatómicamente y estudiados osteométricamente. Se discriminó entre camélidos grandes y pequeños conforme a los grupos de tamaño que comúnmente se emplean para clasificar a los restos óseos de camélidos de contextos arqueológicos (e.g., Mengoni Goñalons y Yacobaccio 2006). Los primeros estarían integrados por el guanaco y la llama, y el segundo por la vicuña y alpaca, aunque aún no se ha identificado a este último morfotipo en tiempos pre-coloniales en el NOA.

El estándar de referencia empleado para dividir entre estos dos grandes grupos fue el guanaco (Lama guanicoe) existente en la región. Se emplearon las medidas relevadas en dos ejemplares actuales de Cumbres Calchaquíes y Nevados del Aconquija (NOA). El gradiente de tamaño considerado para este estudio fue de menor a mayor: vicuña, 
alpaca, guanaco (NOA), llama. Asimismo, trabajos recientes han mostrado que los guanacos del NOA se superponen a las llamas más pequeñas de la misma región (Yacobaccio 20l0). Cabe también contemplarse la presencia de formas híbridas, resultantes del cruzamiento de alpacas y llamas (e.g., huarizos), lo que influiría sobre el tamaño y proporciones anatómicas de esos animales.

Para analizar la variabilidad osteométrica y discriminar entre los diferentes morfotipos se emplearon dos técnicas. Por un lado, se empleó el índice de tamaño logarítmico (LSI) desarrollado por Meadow (1999). Su fórmula es: $d=\log X-$ log estándar $=\log (X / Y)$, dónde $X$ corresponde al espécimen arqueológico representado por una medida en particular. Los valores obtenidos pueden visualizase a través de un gráfico de barras (Figura I) donde se ve claramente a los dos grupos de tamaño principales, a ambos lados del estándar provisto por el guanaco actual del NOA. Además de esta diferenciación en dos grupos principales, se ve la variación total de tamaño de todos los huesos medidos $(n=118)$ en una misma escala, lo que permitió apreciar tres subgrupos: los más pequeños (comparables a vicuñas), los intermedios (posiblemente llamas pequeñas y guanacos) y los más grandes (comparables a llamas grandes).

Esquina de Huajra - Pucará de Volcán - Tolombón

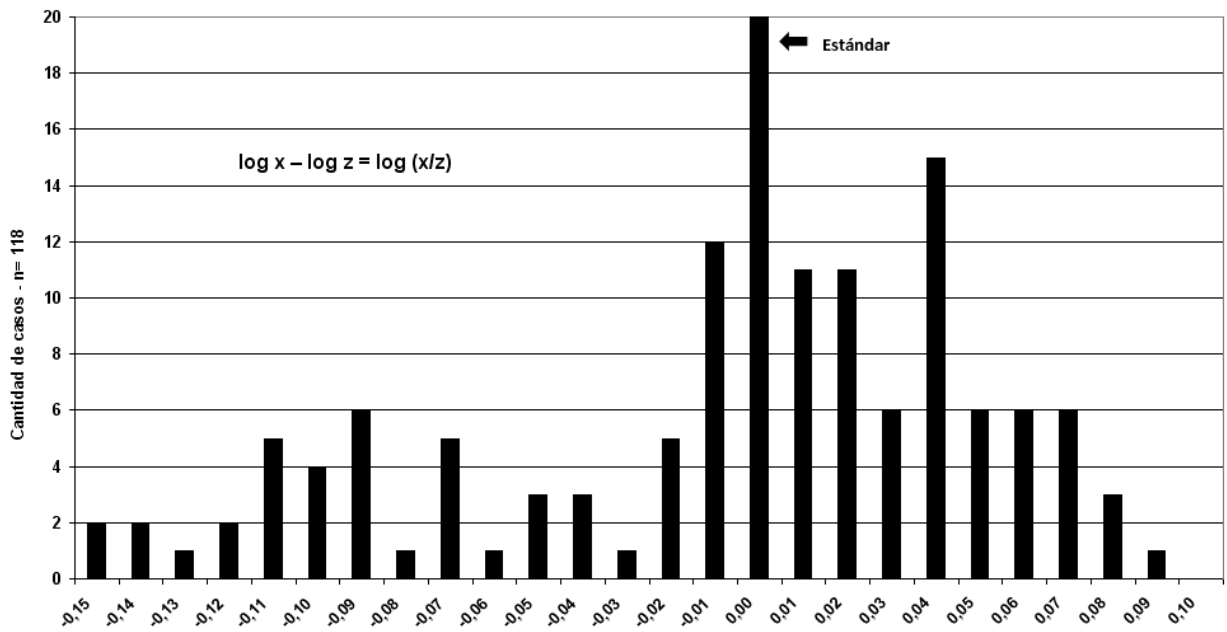

Diferencia logarítimica - Estándar: guanaco NOA

Figura 1: Diferencia logarítmica entre diferentes partes esqueletarias arqueológicas $(\mathrm{n}=118)$ y el estándar moderno (guanaco del NOA).

Figure 1: Log difference between different archaeological skeletal parts and a modern standard.

Adicionalmente, se aplicaron análisis de conglomerados a una muestra de metapodios distales, arqueológicos y actuales (ejemplares de vicuñas de Abra Pampa, Jujuy; los guanacos referidos del NOA; ejemplares de llamas de la Laguna de Pozuelos, Jujuy). Las medidas elegidas fueron: (a) ancho máximo del extremo distal y (b) espesor promedio de ambos cóndilos distales (siguiendo a Kent 1982).

Se probaron diferentes algoritmos (e.g., UPGMA, Ward) para ver si los datos se agrupaban de la misma manera (o no) y así establecer informalmente la robustez de los agrupamientos (Hammer et al. 200I). El dendrograma (Figura 2) muestra dos grupos principales. Un grupo reúne los casos (individuos) más pequeños (vicuñas). El otro grupo incluye dos subgrupos que se corresponderían con los individuos de tamaño más grandes (llamas muy grandes) y un subgrupo intermedio cuyo tamaño sería semejante al guanaco del NOA y al de algunas llamas de la misma región. 


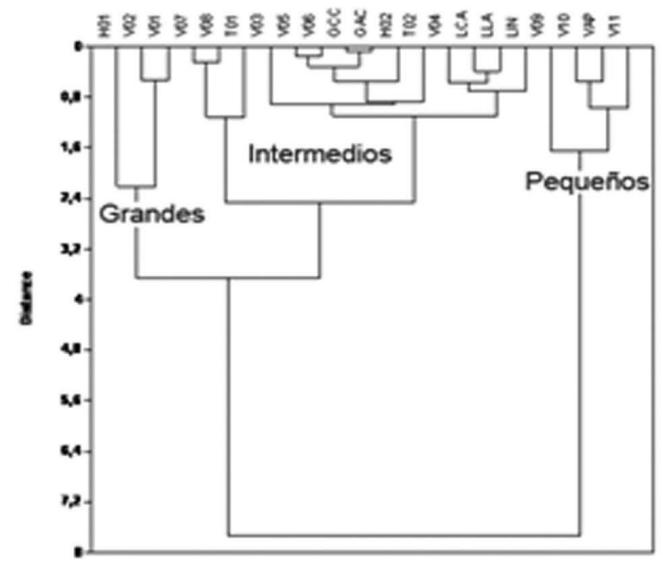

Figura 2: Dendrograma que muestra los agrupamientos de una muestra de metapodios distales. Abreviaturas: casos arqueológicos $(\mathrm{H}=$ E. de Huajra; $\mathrm{V}=$ P. de Volcán; $\mathrm{T}=$ Tolombón) y casos actuales $(\mathrm{GCC}=$ guanaco de Cumbres Calchaquíes; $\mathrm{GAC}=$ guanaco de Nevados de Aconquija; LCA = llama carguera de Laguna de Pozuelos, Jujuy; LLA = llama lanuda de Laguna de Pozuelos, Jujuy; LIN = llama intermedia de Laguna de Pozuelos, Jujuy; VAP = vicuña de Abra Pampa, Jujuy).

Figure 2: Dendogram showing the grouping of a sample of distal metapodials: archaeological ( $H=$ E. de Huajra; $V=P$. de Volcán; $T$ $=$ Tolombón) and present cases of guanacos, llamas and vicuñas.

Estos tres subgrupos fueron empleados como unidades para seleccionar material óseo para los análisis de isótopos. De esa manera podríamos evaluar las variaciones entre los diferentes subgrupos de tamaño y aquellas dentro del interior de cada subgrupo.

\section{ANÁLISIS DE ISÓTOPOSY SUS RESULTADOS}

Se eligieron huesos del esqueleto apendicular de los tres sitios (Pucará de Volcán, $\mathrm{n}$ $=12$; Esquina de Huajra, $\mathrm{n}=5$; Tolombón $\mathrm{n}=\mathrm{II}$ ). En todos los casos se trató de huesos fusionados, pertenecientes a animales mayores de $12-18$ meses (edad mínima). Para esto nos basamos en las secuencias y cronogramas de fusión disponibles para camélidos (e.g., Kent 1982, Kaufmann 2009).

Las muestras fueron analizadas en dos tandas. La primera fue procesada por Randy Culp y Alexander Cherkinsky del Center for Applied Isotope Studies (CAIS) de la University of Georgia y la segunda por Robert Tykot del Department of Anthropology, University of South Florida. En ambos casos los análisis se realizaron sobre colágeno.

Las muestras recibieron un pretratamiento en los respectivos laboratorios para eliminar contaminantes de diferente índole. Para controlar que los valores isotópicos fueran confiables se midió en el laboratorio la concentración de $\mathrm{C}$ (C\%) y $\mathrm{N}(\mathrm{N} \%)$ y se calculó la razón $\mathrm{C} / \mathrm{N}$. Los resultados obtenidos (Tabla I) caen dentro del rango de valores aceptables (2.8 y 3.6) en la literatura especializada (Pate 1994), al igual que las concentraciones de $C$ y $N$ (Ambrose 1990), por lo que se considera que todas las muestras aquí discutidas son igualmente confiables.

El rango de variación del carbono $\left(\delta^{13} \mathrm{C}\right)$ es muy alto cuando se consideran de manera conjunta todas las muestras analizadas (Tabla I). Sus valores van de $-19,6$ a $-8,9 \%$ o (CV = 17,8\%). Esta variación general en los valores isotópicos del carbono también se observa cuando se analizan los resultados tomando en cuenta los tres subgrupos de tamaño identificados, tal como veremos seguidamente (Figura 3). El isótopo de nitrógeno también es muy variable (Tabla I), oscilando entre 2,5 y II,8 \% (CV: $39,3 \%$ ), aunque el promedio se corresponde con lo esperado para mamíferos terrestres (e.g., Pate 1994), diferentes factores podrían estar influyendo sobre estos valores (véase más adelante).

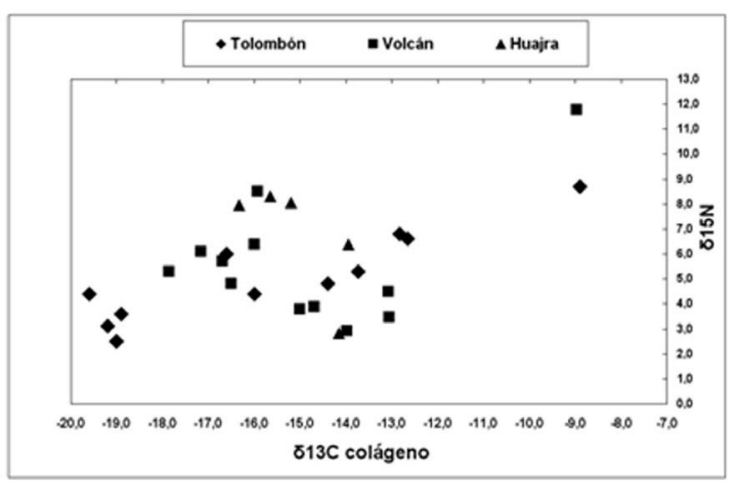

Figura 3: Isótopos de carbono y nitrógeno de las muestras arqueológicas analizadas.

Figure 3: Carbon and nitrogen isotopes of the archaeological samples.

El subgrupo de tamaño más pequeño $(\mathrm{n}=8)$ tiene valores $\delta^{13} \mathrm{C}$ cuya variación (-19,2\%。 a I4,2\%。) es menor a la de los otros subgrupos $(\mathrm{CV}=1 \mathrm{I}, 6 \%)$. En 
este grupo hay ejemplares cuyo tamaño es comparable a la vicuña actual. Estos últimos individuos muestran diferencias en el carbono dependiendo del sitio del que provienen. Los individuos de Tolombón tienen una firma isotópica que se superpone a la de las vicuñas que viven a más de 4000 m, mientras que los de P. deVolcán poseen valores más positivos que se corresponden con los de aquellas vicuñas que hoy viven a menos de $4000 \mathrm{~m}$. Dentro de este último subgrupo caen algunos individuos de P. de Volcán y E. de Huajra que no son tan pequeños como los anteriores, pero que igualmente tienen una dieta más enriquecida.

\begin{tabular}{|c|c|c|c|c|c|c|c|}
\hline Muestra & Hueso & Porción & $\log (x / g)$ & Subgrupo de Tamaño & $\delta^{13} \mathbf{C}$ & $\delta^{15} \mathbf{N}$ & $\mathbf{C} / \mathbf{N}$ \\
\hline $\mathrm{H}-26 \mathrm{~A}$ & Tibia & $\mathrm{px}$ & $-0,07$ & Pequeño & $-14,2$ & 2,8 & 2,9 \\
\hline $\mathrm{H}-26 \mathrm{C}$ & Metapodio & ds & $-0,01$ & Intermedio & $-15,7$ & 8,3 & 2,9 \\
\hline $\mathrm{H}-26 \mathrm{~B}$ & Radioulna & ds & 0,02 & Intermedio & $-16,3$ & 8,0 & 2,9 \\
\hline $\mathrm{H}-25 \mathrm{~A}$ & Metapodio & ds & 0,05 & Grande & $-15,2$ & 8,1 & 2,9 \\
\hline$H-26 D$ & Escápula & $\mathrm{px}$ & 0,08 & Grande & $-13,9$ & 6,4 & 2,9 \\
\hline V-82A & Metapodio & ds & $-0,15$ & Pequeño & $-17,9$ & 5,3 & 2,9 \\
\hline V-827 & Metapodio & ds & $-0,14$ & Pequeño & $-16,7$ & 5,7 & 3,3 \\
\hline$V-72 A$ & Radioulna & ds & $-0,11$ & Pequeño & $-15,9$ & 8,5 & 2,9 \\
\hline$V-524$ & Metapodio & ds & $-0,04$ & Pequeño & $-14,7$ & 3,9 & 3,3 \\
\hline$V-42 B$ & Húmero & ds & $-0,01$ & Intermedio & $-14,0$ & 2,9 & 2,9 \\
\hline$V-62 A$ & Metatarso & $\mathrm{px}$ & 0,01 & Intermedio & $-|3|$, & 3,5 & 2,9 \\
\hline$V-82 B$ & Metatarso & $\mathrm{px}$ & 0,02 & Intermedio & $-17,2$ & $6, I$ & 2,9 \\
\hline$V-2 A A$ & Escápula & $\mathrm{px}$ & 0,04 & Grande & $-15,0$ & 3,8 & 3,4 \\
\hline$V-625$ & Radioulna & ds & 0,04 & Grande & $-16,0$ & 6,4 & 3,2 \\
\hline$V-3 B B$ & Radioulna & ds & 0,04 & Grande & $-16,5$ & 4,8 & 3,1 \\
\hline$V-32 A$ & Metatarso & $\mathrm{px}$ & 0,06 & Grande & $-9,0$ & 11,8 & 3,0 \\
\hline$V-32 B$ & Tibia & ds & 0,08 & Grande & $-|3|$, & 4,5 & 2,8 \\
\hline $\mathrm{T}-430$ & Falange I & co & $-0,11$ & Pequeño & $-19,2$ & 3,1 & 3,1 \\
\hline T-199 & Escápula & ds & $-0,07$ & Pequeño & $-19,0$ & 2,5 & 2,9 \\
\hline $\mathrm{T}-155$ & Falange I & co & $-0,05$ & Pequeño & $-18,9$ & 3,6 & 3,3 \\
\hline $\mathrm{T}-123$ & Metapodio & ds & $-0,02$ & Intermedio & $-12,7$ & 6,6 & 2,8 \\
\hline $\mathrm{T}-574$ & Metapodio & ds & 0,01 & Intermedio & $-13,7$ & 5,3 & 2,8 \\
\hline $\mathrm{T}-485$ & Metacarpo & $\mathrm{px}$ & 0,02 & Intermedio & $-16,6$ & 6,0 & 3,3 \\
\hline $\mathrm{T}-577$ & Radioulna & ds & 0,02 & Intermedio & $-8,9$ & 8,7 & 3,2 \\
\hline T-58I & Calcáneo & co & 0,02 & Intermedio & $-16,0$ & 4,4 & 3,2 \\
\hline T-629 & Radioulna & $\mathrm{px}$ & 0,04 & Grande & $-12,8$ & 6,8 & 2,9 \\
\hline T-47I & Falange I & co & 0,05 & Grande & $-19,6$ & 4,4 & 3,3 \\
\hline $\mathrm{T}-402$ & Metapodio & ds & 0,09 & Grande & $-14,4$ & 4,8 & 3,3 \\
\hline
\end{tabular}

Tabla 1: Materiales óseos analizados. Abreviaturas: $\mathrm{H}=$ Esquina de Huajra; $\mathrm{V}=$ Pucará de Volcán; $\mathrm{T}=$ Tolombón; co = completo; $\mathrm{px}=$ proximal; ds = distal.

Table 1: Bone materials analized: $H=$ Esquina de Huajra $V=$ Pucará de Volcán; $T=$ Tolombón; $c o=$ completo; $p x=$ proximal; $d s=$ distal .

El grupo de tamaño intermedio $(n=10)$ es más variable $(-17,2 \%$ a $-8,9 \%$ ) que el anterior $(\mathrm{CV}=$ 17,3\%). Las dietas de los animales se agrupan en tres subconjuntos. En todos los casos los valores se alejan de los documentados para guanaco del NOA (ver valor de referencia más arriba). Algunos individuos con valores muy negativos se superponen a los valores actuales de las llamas que pastan por debajo 
de los $4.000 \mathrm{~m}$. Otros con valores intermedios son algo más positivos, indicando una dieta mixta $\mathrm{C}_{3}$ y $\mathrm{C}_{4}$. Un caso aislado muestra una firma muy enriquecida lo que sugiere una dieta basada en plantas $\mathrm{C}_{4}$.

El subgrupo grande $(\mathrm{n}=10)$ es el más variable $(-19,6 \%$ a $-9,0 \%$; $C V=19,2 \%)$. Hay un individuo de Tolombón que tiene una firma isotópica para el carbono muy negativa, comparable al de algunas llamas actuales que viven a más de $4000 \mathrm{~m}$ de altura. Los ejemplares restantes de esta localidad y la mayoría de los individuos de P. de Volcán presentan valores más enriquecidos, al igual que los de $\mathrm{E}$. de Huajra. Además, un individuo de Volcán posee una firma muy positiva que se corresponde con valores altos del nitrógeno (Tabla I).

Los valores de $\delta^{15} \mathrm{~N}$ son variables (Tabla I) pero caen dentro de los esperados para herbívoros que son bebedores obligados (e.g, vicuña) o periódicos (e.g., guanaco), siendo los valores de los primeros más bajos que los de los segundos (Ambrose 1991).Además, estas variaciones se relacionarían con aspectos de la fisiología de los animales que están actualmente en estudio experimental (Sponheimer et al. 2003). Asimismo, los valores altos de este isótopo observados en algunos casos podrían relacionarse con el pastoreo en campos fertilizados, tal como lo han mostrado estudios recientes (Szpak et al. 20I2)

\section{DISCUSIÓN GENERAL}

En Esquina de Huajra $(n=5)$ la dieta de los camélidos consumidos en el sitio habría sido relativamente homogénea (Tabla I; Figura 3), con valores para el $\delta^{13} \mathrm{C}$ entre $-16,3 \%$ y $-13,9 \%$ (CV $=$ $6,7 \%$ ). Esta variación es independiente del subgrupo de tamaño al que pertenecen los individuos analizados. Los valores de carbono son muy semejantes a los de los camélidos actuales que pastan a menos de 4000 $\mathrm{m}$. Esto significa que tanto los animales domesticados (llamas) como los silvestres (vicuñas) tuvieron acceso a pasturas de semejante composición integradas por plantas C3 y C4.

En Pucará de Volcán $(\mathrm{n}=12)$ los tres subgrupos de tamaño están representados por animales cuya firma isotópica $\left(\delta^{13} \mathrm{C}\right)$ es muy variable $(C V=16,2 \%)$, con valores entre $-17,9 \%$ y $-9,0 \%$ 。 (Tabla I; Figura 3), aunque en la mayoría de los casos se superponen a los valores de los camélidos actuales (llama y vicuña) que viven por debajo de los $4000 \mathrm{~m}$. Pese a ello el subgrupo de tamaño intermedio y grande cuenta con individuos con dieta enriquecida en carbono ( $>-13,0 \%$ ). Esto sugiere que los animales que podrían caer dentro del rango de tamaño de las llamas habrían tenido acceso a pasturas con diferente composición. Esto plantea la existencia de hatos segregados cuya alimentación diferencial podría relacionarse con que la localidad era abastecida desde diversas zonas altitudinales $y$ por ende, compatible con la existencia de complejas redes de redistribución de estos productos y bienes.

Las muestras de Tolombón $(\mathrm{n}=\mathrm{II})$ presentan una interesante variabilidad ( $C V=21,8 \%)$, con valores isotópicos para el $\delta^{13} \mathrm{C}$ entre $-19,6 \%$ y $-8,9 \%$ (Tabla I; Figura 3 ). Esto sugiere que los animales representados por estas muestras se alimentaron de plantas con firma isotópica marcadamente diferente. Hay individuos muy pequeños (vicuñas) que tienen una dieta semejante a las vicuñas que viven a más de $4.000 \mathrm{~m}$ de altitud. Los ejemplares del subgrupo intermedio muestran gran variabilidad, en general, con dietas comparables a las de los camélidos (llamas) que viven por debajo de los $4.000 \mathrm{~m}$. Un individuo particular tiene una dieta muy enriquecida que sugiere una dieta centrada en plantas $\mathrm{C}_{4}$. Los individuos de mayor tamaño poseen para el $\delta^{13} \mathrm{C}$ valores mucho más variables que se superponen tanto a las llamas que pastan por encima como a aquellas que viven por debajo de los $4000 \mathrm{~m}$. Esto sugiere la cría de camélidos en ambientes que poseían pasturas de composición $\left(C_{3}\right.$ y $\left.C_{4}\right)$ disímil, sugiriendo que en esta localidad se disponía de recursos animales que pastaban en lugares distantes unos de otros, y se accedía a recursos segregados espacialmente a través de un sistema de redistribución.

\section{CONSIDERACIONES FINALES}

Al comparar los agrupamientos obtenidos por la osteometría y aquellos provistos por los isótopos, no siempre se da una correspondencia, lo que era esperable dada la variabilidad observada en la dieta de los camélidos actuales, independiente de su categoría taxonómica. Animales del mismo tamaño poseen diferente dieta dependiendo del contexto ambiental donde se alimentaron. En algunos casos 
estas diferencias sugieren la existencia de hatos segregados, lo que indica un notorio control sobre la producción de estos animales, en el caso de los domesticados (llamas). Asimismo, las diferencias en las dietas de los animales de tamaño más pequeños (vicuñas) apuntan hacia la existencia de zonas de aprovisionamiento distintas $y$ de relativamente extensas redes de circulación de productos empleados en la alimentación humana.

Un aspecto que debe tenerse en cuenta para evaluar el alcance de estos resultados es el tipo de depósito y contexto arqueológico del que provienen las muestras presentadas. En Esquina de Huajra los huesos analizados provienen de un espacio doméstico (patio) adyacente a una vivienda (Cremonte y Gheggi 20I2). Estos se asocian con elementos necesarios para la preparación y almacenamiento de los alimentos y con vajilla doméstica (pucos, escudillas, platos, fuentes) de prestigio empleada como servicio de mesa (Scaro y Cremonte 2012). El análisis arqueofaunístico integral de estos conjuntos también sugiere la preparación y consumo de partes anatómicas de camélidos jóvenes y adultos-jóvenes (Mengoni Goñalons 2013).

En Pucará de Volcán el material faunístico proviene de un basural general que se asocia a una serie de recintos y espacios abiertos que rodean a un gran montículo artificial (Garay de Fumagalli 1998). Esto sugiere que estamos ante un depósito más complejo, que seguramente captó el descarte y limpieza de diferentes sectores del asentamiento. Por ende, allí es esperable una mayor variabilidad que en los otros tipos de depósitos. El consumo estuvo centrado en el acceso preferencial a animales en edad óptima como productores de carne (2-3 años), lo que sugiere una notoria selectividad en los sacrificios (Mengoni Goñalons 20l3).

En Tolombón los materiales óseos provienen de un espacio doméstico de un recinto habitacional (Williams et al. 2013), en relación con bienes de prestigio y cerámica culinaria asociada a una estructura de fogón que persiste a lo largo de varios niveles artificiales. De esto se desprende que los restos óseos derivarían de tareas cotidianas asociadas con la preparación de las comidas, en las que la carne de camélidos entró como ingrediente importante. Los perfiles etarios sugieren la disponibilidad de un excedente en la producción.
También se tuvo acceso a recursos de caza como la vicuña (Mengoni Goñalons 20l3).

Basados en la información presentada es posible plantear diferencias en la complejidad de los mecanismos de producción, distribución y consumo final de los alimentos de origen animal, teniendo en cuenta el grado de control ejercido sobre los rebaños silvestres y domesticados. En Esquina de Huajra los animales presentes se corresponderían con planteles que vivieron en pastizales de baja altura $(<4.000$ $m$ ). En Pucará del Volcán la variabilidad observada es compatible con formas de manejo que implicaron la segregación de hatos de animales domesticados que pastorearon en diferentes campos de pasturas y la obtención de animales silvestres de zonas relativamente cercanas. En Tolombón, los animales domesticados habrían sido criados en zonas de más baja altitud $(<4.000 \mathrm{~m}$ ) y los silvestres habrían venido de más altura y de cierta distancia.

En síntesis, hemos visto que los isótopos estables constituyen una herramienta útil para discutir diferentes aspectos relacionados con el uso y manejo de los camélidos. El potencial de estos tipos de análisis complementa y enriquece los estudios anatómicos, taxonómicos, osteométricos o paleopatológicos. Pensamos que esa complementariedad marca una línea de trabajo que es preciso ampliar, desarrollar y profundizar.

Agradecimientos: Los análisis de isótopos fueron realizados con el PICT-I4425 dirigido por Verónica I. Williams y el PIP- 536I codirigido por el autor. A Andrés Izeta del Museo de Antropología de Córdoba por facilitarme las medidas del guanaco de Nevados del Aconquija. A Dolores Elkin con quien relevamos el guanaco de Cumbres Calchaquíes. A Alexander Cherkinsky, Randy Culp y Robert Tykot por sus sugerencias y asesoramiento. Cualquier error de interpretación incurrido en este trabajo es de mi propia responsabilidad.

\section{BIBLIOGRAFÍA}

Ambrose, S.H. 1990. "Preparation and characterization of bone and tooth collagen for stable carbon and nitrogen isotope analysis". Journal of Archaeological Science 17:430-45I.

Ambrose, S. H. 1991. "Efects of diet, climate and physiology on nitrogen isotope abundance in terrestrial foodwebs". Journal of Archaeological Science 18: 293-317. 
Cremonte, M. B., S. Gheggi. 2012. "Espacios rituales y cultura material en un sitio arqueológico Humahuaca-Inca (Quebrada de Humahuaca, Jujuy, Argentina)”. Revista Española de Antropología Americana 42(I): 9-27.

Dantas, M. 2012. "Identificación interespecífica de camélidos en el valle de Ambato (Catamarca, Argentina). Una aproximación a la problemática desde distintas líneas de análisis". Revista del Museo de Antropología 5: 259-268.

Fernández, J. y H. O Panarello 1999-200I."Isótopos del carbono en la dieta de herbívoros y carnívoros de los Andes jujeños”. XAMA I2-|4: 7|-85.

Garay De Fumagalli, M. 1998. "El Pucara de Volcán: historia ocupacional y patrón de instalación”. En Los desarrollos locales y sus territorios, compilado por B. Cremonte, pp: I3|-|53. Universidad Nacional de Jujuy, Jujuy.

Hammer, Ø., Harper, D.A.T., Ryan, P.D. $200 \mathrm{I}$ "PAST: Paleontological statistics software package for education and data analysis". Palaeontologia Electronica 4(I): 9pp. http://palaeoelectronica.org/200I_I/past/issuel_0I.htm.

Izeta, A. D., A. G. Laguens, M. B. Marconetto y M. C. Scattolin. 2009. "Camelid handling in the meridional Andes during the first millennium AD: a preliminary approach using stable isotopes". International Journal of Osteoarchaeology, 19(2): 204-2।4.

Kaufmann, C. 2009. Estructura de Edad y Sexo en Guanaco. Sociedad Argentina de Antropología, Buenos Aires.

Kent, J. D. 1982. The domestication and exploitation of the South American camelids: Methods of analysis and their application to circum-lacustrine archaeological sites in Bolivia and Peru.Tesis de Ph.D. Washington University, St. Louis.

López, P. I. Cartajena, B. Santander y B. Rivera. 20I2. "Análisis de isótopos estables $(\Delta \mid 3 c$ y $\Delta \mid 5 n)$ en camélidos silvestres y domésticos delValle Del Mauro (IV Región, Chile): una visión general desde el Arcaico Tardío hasta el Período Tardío". Trabajo presentado en el XIX Congreso Nacional de Arqueología Chilena, Arica.

Meadow, R. H. 1999. "The use of size index scaling techniques for research on archaeozoological collections from the Middle East". En Historia Animalium ex Ossibus, Festschrift für Angela von den Driesch, editado por C. Becker, H. Manhart, J. Peters J.y J. Schibler, pp. 285-300.Verlag Marie Leidorf GmbH, Rahden/Westf.

Mengoni Goñalons, G. L. 2007. “Camelid management during Inca times in NW Argentina: models and archaeological indicators". Anthropozoologica 42 (2): |29-|4|.

2013. "El aprovechamiento de la fauna en sociedades complejas del NOA: aspectos metodológicos y su aplicación en diferentes contextos arqueológicos". En Al borde del imperio. Paisajes sociales, materialidad y memoria en áreas periféricas del noroeste argentino, editado por V. Williams y B. Cremonte, Pp. 3 I I-343. Sociedad Argentina de Antropología, Buenos Aires.

Mengoni Goñalons, G. L. y H. D. Yacobaccio. 2006. "The domestication of South American camelids: a view from the South-Central Andes". En Documenting Domestication: New Genetic and Archaeological Paradigms, editado por M. A. Zeder, D. Bradley, E. Emshwiller y B. D. Smith, pp. 228-244. University of California Press, Berkeley.

Panarello, H.O. y J. Fernández 2002 "Stable carbon isotope measurements on hair from wild animals from altiplanic environments of Jujuy". Radiocarbon 44:709-7I6.

Pate, F.D. 1994."Bone chemistry and paleodiet".Journal of Archaeological Method and Theory I: 161-209.

Scaro, A. y M.B. Cremonte. 20/2. "La vajilla de servicio de Esquina de Huajra (Dpto. Tumbaya, Jujuy, Argentina). Alternativas teóricas para interpretar su significado". Revista del Museo de Antropología 5: 3I-44.

Szpak, P., J.F. Millaire, C. D. White, F.J. Longstaffe. 2012."Influence of seabird guano and camelid dung fertilization on the nitrogen isotopic composition of field-grown maize (Zea mays)". Journal of Archaeological Science 39: 372 I-3740.

Sponheimer, M., T. Robinson, B. Roeder, J. Hammer, L. Ayliffe, B. Passey, T. Cerling, D. Dearing y J. Ehleringer. 2003. "Digestion and passage rates of grass hays by llamas, alpacas, goats, rabbits, and horses". Small Ruminant Research 48: I49-I54.

Thornton, E.K., S. De France, J. Krigbaum y P.R. Williams. 2010. "Isotopic evidence of Middle Horizon to $16^{\text {th }}$ century camelid herding in the Osmore valley, Peru". International Journal of Osteoarchaeology 2I(5): 544-567.

Williams, V. I., M.P. Villegas y L. Arechaga 2013. "Paisajes de Tolombón. ¿De poblado tardío a capital de un wamani?" En Al borde del imperio. Paisajes sociales, materialidad y memoria en áreas periféricas del noroeste argentino, editado por V. Williams y B. Cremonte, pp. 143-176. Sociedad Argentina de Antropología, Buenos Aires.

Yacobaccio, H. D. 2010. "Osteometría de llamas (Lama glama L.) y sus consecuencias arqueológicas”. En Zooarqueología a principios del siglo XXI, editado por M. Gutiérrez, M. De Nigris, P. Fernández, M. Giardina,A. Gil,A. Izeta, G. Neme y H.Yacobaccio, pp. 65-75. Ediciones Libros del Espinillo, Buenos Aires.

Yacobaccio, H.D., C. T. Samec y M.P. Catá. 2010. "Isótopos estables y zooarqueologia de camélidos en contextos pastoriles de la puna (Jujuy, Argentina)". En Zooarqueología a principios del siglo XXI, editado por M. Gutiérrez, M. De Nigris, P. Fernández, M. Giardina,A. Gil,A. Izeta, G. Neme y H.Yacobaccio pp. 77-86. Ediciones Libros del Espinillo, Buenos Aires. 